ARTIGO ORIGINAL

ORIGINAL ARTICLE

Palavras-chave:

NGS, CPNPC, custo efetividade

Keywords:

NGS, NSCLC, cost-effectiveness

\section{Análise de custo-efetividade do painel de sequenciamento de nova geração do DNA circulante tumoral no diagnóstico dos pacientes com câncer de pulmão de não pequenas células não escamoso metastático}

\author{
Cost-effectiveness analysis of next generation sequencing \\ panel of circulating tumor DNA in the diagnosis of patient \\ with metastatic non-squamous non-small cell lung cancer
}

Luiz Henrique de Lima Araujo', Renata Coudry², Clarissa Seródio Baldotto1,3,

Mariana Mioti Sebastião ${ }^{4}$, Marisa D'Innocenzo ${ }^{4}$, Micha Nussbaum ${ }^{4}$ Rodrigo Shimabukuro Ho ${ }^{4}$

DOI: 10.21115/JBES.v11.n3.p221-30

\section{RESUMO}

Objetivo: Avaliar o impacto clínico e econômico do uso do perfil genômico utilizando Next Generation Sequencing (NGS) em DNA circulante tumoral (ctDNA) na escolha do tratamento de primeira linha (1L) dos pacientes com câncer de pulmão de células não pequenas, não escamoso, metastático e que não apresentam material tecidual suficiente para avaliação das mutações oncogênicas. Métodos: Foi realizada uma análise de custo-efetividade com base em um modelo de árvore de decisão e um modelo de Markov para simular os resultados dos testes diagnósticos e consequentemente o seu impacto clínico e econômico na primeira linha de tratamento. O comparador da análise foi o teste de mutações específicas no gene EGFR por ctDNA. As terapias medicamentosas incluídas na análise foram as terapias-alvo de EGFR e ALK, que estão incorporadas no rol da Agência Nacional de Saúde Suplementar, e a imunoterapia pembrolizumabe combinada à quimioterapia. Os desfechos clínicos foram retirados dos estudos clínicos das terapias avaliadas no modelo. Resultados: $\mathrm{O}$ uso do painel de NGS em ctDNA demonstrou uma economia de -R\$2.076,35 por paciente em um ano, e os resultados de RCEl foram: -R\$ 7.652,56 (R\$/SLP) e -R\$33.742,14 (R\$/SG). Conclusão: O painel de NGS em ctDNA demonstrou ser uma alternativa dominante em relação ao teste de EGFR em ctDNA.

\begin{abstract}
Objective: The aim of this study was to evaluate the clinical and economic impact of the next generation sequencing (NGS) panel of circulating tumor DNA (ctDNA) in the clinical decision of first line treatment for patients with metastatic non-squamous non-small cell lung cancer who lack of tissue material for evaluation of oncogenic driver mutations. Methods: A cost-effectiveness analysis was performed based on a decision tree model and a Markov model in order to simulate the results of diagnostic tests and therefore its clinical and economic impact in the first line of treatment. The comparators were the single EGFR mutation detection methodologies in ctDNA. The analysis included the anti-EGFR and anti-ALK target therapies; and the combined therapy of pembrolizumab plus chemotherapy. Clinical outcomes were derived from clinical trials of the therapies included in the model. Results: The use of the NGS ctDNA panel showed a saving of -R\$2,076.35 and the results of the ICER were -R\$7,652.56 (R\$/SLP) and -R\$33,742.14 (R\$/SG). Conclusion: The NGS panel demonstrated to be a dominant alternative in comparison to ctDNA EGFR testing.
\end{abstract}

Recebido em: 30/10/2019. Aprovado para publicação em: 13/11/2019

1. Grupo Brasileiro de Oncologia Torácica, Porto Alegre, RS, Brasil.

2. UnitedHealth Group, Minnetonka, Minnesota, EUA.

3. Instituto D'Or de Pesquisa e Ensino, São Paulo, SP, Brasil.

4. Roche Diagnóstica Brasil, São Paulo, SP, Brasil.

Instituição onde o trabalho foi executado: Roche Diagnóstica do Brasil Ltda.

Financiamento: Este estudo não teve financiamento.

Autor correspondente: Rodrigo Shimabukuro Ho. Av. Engenheiro Billings, 1729, Jaguaré, São Paulo, SP. CEP: 05321-900.

E-mail: rodrigo.ho@roche.com 


\section{Introdução}

O câncer de pulmão é o câncer de maior incidência no mundo (11,6\% de todos os casos), além de representar a principal causa de morte por câncer (Bray et al., 2018). No Brasil, de acordo com o Instituto Nacional do Câncer (Inca), foram estimados 31.270 novos casos de câncer de pulmão em 2018, sendo $18.740 \mathrm{em}$ homens e $12.530 \mathrm{em}$ mulheres, o que corresponde a $8,7 \%$ e $6,2 \%$ de todos os casos de câncer no país, respectivamente (Inca, 2017).

A classificação da Organização Mundial da Saúde (OMS) para o câncer de pulmão primário reconhece quatro principais tipos histológicos: câncer de pulmão de pequenas células (CPPC), adenocarcinoma, carcinoma de células escamosas e carcinoma de células grandes; os três últimos tipos são conhecidos como carcinomas de células não pequenas (CPNPCs) (Brambilla et al., 2001). Entre os tipos histológicos, o CPNPC é o mais comum, representando $85 \%$ de todos os casos (Barros et al., 2006; Navada et al., 2006; Sher et al., 2008). Para fins práticos, o CPNPC é comumente dividido em dois tipos histológicos principais: carcinoma de células escamosas e carcinoma de células não escamosas (Travis et al., 2011). O carcinoma de células não escamosas representa aproximadamente 80\% do CPNPC (Baldotto et al., 2018).

O CPNPC é considerado uma doença heterogênea com diversas características moleculares (Chouaid et al., 2014). Na era da medicina personalizada, a classificação histológica e a caracterização molecular são fundamentais para a decisão terapêutica (Travis et al., 2013). O National Comprehensive Cancer Network (NCCN) recomenda que os pacientes com CPNPC metastático sejam testados para avaliar a presença de alterações genômicas (mutação ou fusão) nos genes EGFR, ALK, ROS-1, BRAF e NTRK, e para avaliar a expressão de PD-L1. O NCCN aconselha que o teste seja realizado, sempre que possível, com uma avaliação ampla do perfil molecular do tumor, com o intuito de identificar as mutações ativadoras menos frequentes, para as quais os medicamentos eficazes possam estar disponíveis, dentro ou fora de estudos clínicos (National Comprehensive Cancer Network, 2019).

No Brasil, a maioria dos pacientes com CPNPC é diagnosticada em estádio avançado ou irressecável. Geralmente, a investigação é realizada por meio de procedimentos de broncoscopia com biópsia (endobrônquicas ou transbrônquicas) ou biópsia por agulha guiada por tomografia computadorizada (Baldotto et al., 2018). A biópsia tecidual é o padrão-ouro para a realização dos testes moleculares, porém a baixa qualidade e a quantidade inadequada de tecido obtido podem tornar os testes para avaliação das mutações oncogênicas inexequíveis (Liu et al., 2018). Estudos têm demonstrado que aproximadamente $20 \%$ a $30 \%$ dos pacientes com CPNPC apresentam falta de material tecidual para avaliação das mutações oncogênicas (Thompson et al., 2016; Liu et al., 2018).
Para casos em que o material tecidual é insuficiente ou inacessível, a avaliação de alterações moleculares utilizando DNA tumoral circulante (ctDNA), também chamada de biópsia líquida, é o procedimento recomendado pelo International Association for the Study of Lung Cancer (IASLC) para orientação do tratamento clínico dos pacientes com CPNPC avançado (Rolfo et al., 2018).

O ctDNA no sangue é derivado de células tumorais e é, geralmente, liberado por células apoptóticas ou necróticas, ou pela destruição de células tumorais circulantes (CTCS) por apoptose, sistema imunológico ou anoikis. O ctDNA é considerado uma fração do DNA total livre circulante (cfDNA) derivado de eventos de remodelação fisiológica dos tecidos (Hong \& Zu, 2013; Dive \& Brady, 2017; Han et al., 2017). A variabilidade da abundância de ctDNA está associada a carga tumoral, estádio da doença, vascularização, renovação celular e resposta à terapia (Diehl et al., 2008). As alterações no ctDNA podem ser avaliadas por uma variedade de técnicas, incluindo reação em cadeia da polimerase (PCR) alelo-específica, PCR digital e sequenciamento de segunda geração (NGS) (Han et al., 2017).

Os painéis de NGS são plataformas confiáveis e preferíveis por conseguirem detectar, além de mutações comuns, outras alterações com acurácia como: inserções, deleções, alterações no número de cópias e translocações (Plagnol et al., 2018). Os painéis de NGS podem alcançar níveis aceitáveis de sensibilidade e níveis ótimos de especificidade, inclusive no caso de biópsia líquida (Müller et al., 2017).

A IASLC recomenda que seja iniciado o tratamento de primeira linha nos casos em que o NGS em ctDNA seja positivo para alterações nos genes EGFR, ALK, ROS-1 ou BRAF (Rolfo et al., 2018).

O objetivo do presente estudo foi avaliar a relação de custo-efetividade do painel de NGS em ctDNA (NGS ctDNA) versus teste de mutações específicas no gene EGFR em ctDNA (EGFR ctDNA) na escolha do tratamento oncológico de primeira linha (1L) dos pacientes com CPNPC não escamoso, metastático e que não apresentam material tecidual suficiente para avaliação das mutações oncogênicas.

O estudo buscou representar a perspectiva do Sistema Suplementar de Saúde (SSS). O teste EGFR ctDNA é o único marcador disponível para biópsia líquida que apresenta terapia-alvo na lista dos medicamentos antineoplásicos orais com cobertura obrigatória no SSS (Tabela 1).

\section{Métodos}

O estudo foi conduzido com o intuito de estimar o impacto clínico e econômico da detecção de alterações nos genes EGFR e ALK pelo painel de NGS em ctDNA versus teste de mutações específicas no gene EGFR em ctDNA.

\section{Dados de acurácia}

Os dados de acurácia do EGFR ctDNA foram retirados de uma metanálise que avaliou diversos métodos de deteç̧ão do EGFR em ctDNA. As tecnologias consideradas no estudo estão descritas na Tabela 2. 
Tabela 1. Relação das terapias de alvo molecular aprovadas e o rol de cobertura para CPNPC

\begin{tabular}{|c|c|c|}
\hline Biomarcadores & Terapias & $\begin{array}{l}\text { Rol (Agência Nacional de } \\
\text { Saúde Suplementar, 2018) }\end{array}$ \\
\hline EGFR & Erlotinibe & $\operatorname{Sim}^{*}$ \\
\hline EGFR & Afatinibe & Sim + \\
\hline EGFR & Gefitinibe & $\operatorname{Sim}^{*}$ \\
\hline EGFR & Osimertinibe & Não \\
\hline$A L K$ & Crizotinibe & Sim \\
\hline$A L K$ & Alectinibe & Não \\
\hline ROS1 & Crizotinine & Não \\
\hline BRAF & Dabrafenibe + Trametinibe & Não \\
\hline NTRK & Larotrectinibe & Não \\
\hline
\end{tabular}

* Pacientes com CPNPC não escamoso; + pacientes com CPNPC com histologia de adenocarcinoma.

Tabela 2. Metodologias avaliadas na metanálise (Qiu et al., 2015)

\begin{tabular}{|c|c|c|c|c|c|}
\hline Estudo & Métodos de detecção & TP & FP & FN & $\mathrm{TN}$ \\
\hline Siriam KB & ME-PCR & 3 & 0 & 3 & 58 \\
\hline $\mathrm{He} \mathrm{C}$ & ME-PCR & 8 & 0 & 1 & 9 \\
\hline Young TK & Digital PCR & 11 & 1 & 0 & 17 \\
\hline Jiang B & Mutant-enriched sequencing & 14 & 0 & 4 & 40 \\
\hline $\mathrm{HuC}$ & HRM & 22 & 2 & 0 & 0 \\
\hline Huang Z & DHPLC & 188 & 81 & 108 & 445 \\
\hline XuF & ARMS & 4 & 0 & 4 & 26 \\
\hline Yam I & AS-APEX & 30 & 1 & 0 & 4 \\
\hline Jing CW & HRM & 29 & 2 & 16 & 73 \\
\hline Liu X & ARMS & 27 & 0 & 13 & 46 \\
\hline LvC & DHPLC & 0 & 0 & 3 & 3 \\
\hline Zhang $\mathrm{H}$ & MEL & 15 & 0 & 7 & 64 \\
\hline Zhao X & ME-PCR & 16 & 3 & 29 & 63 \\
\hline Wang S & ARMS & 15 & 2 & 53 & 64 \\
\hline Kimura H & ARMS & 6 & 1 & 2 & 2 \\
\hline Kimura H & ARMS & 6 & 1 & 2 & 33 \\
\hline Taniguchi K & BEAMing & 32 & 0 & 12 & 0 \\
\hline Goto K & AS-APEX & 22 & 0 & 29 & 35 \\
\hline Nakamura T & Inhibiting PCR-quenching probe method & 21 & 0 & 26 & 23 \\
\hline Kim HR & PNAClamp & 6 & 0 & 29 & 5 \\
\hline Kim ST & PNA-LNA PCR clamp & 8 & 3 & 4 & 42 \\
\hline Kuang Y & ARMS & 21 & 2 & 9 & 11 \\
\hline Brevet M & Sequenom & 8 & 2 & 10 & 11 \\
\hline LiX (plasma) & ARMS & 27 & 3 & 29 & 62 \\
\hline Li X (serum) & ARMS & 19 & 2 & 29 & 42 \\
\hline Douillard JY & ARMS & 69 & 1 & 36 & 546 \\
\hline Weber B & Cobas EGFR blood test & 17 & 6 & 11 & 162 \\
\hline
\end{tabular}

HRM: high-resolution melting; PNA-LNA: peptide nucleic acid-locked nucleic acid; AS-APEX: allele-specific arrayed primer extension; ME-PCR: mutant-enriched-PCR; DHPLC: denaturing high-performance liquid chromatography; BEAMing: beads, emulsion, amplification, and magnetics; ARMS: amplification refractory mutation system; MEL: mutant-enriched liquid chip. 
Até o presente momento, não foi publicada uma metanálise para avaliar a sensibilidade e a especificidade do painel de NGS em ctDNA para pacientes com câncer de pulmão em estádio avançado. Os dados de acurácia do painel de NGS em ctDNA foram retirados de um estudo que avaliou as alterações nos genes EGFR ou ALK nesses pacientes (Paweletz et al., 2016) (Tabela 3).

\section{Modelo}

A análise foi baseada em um modelo de árvore de decisão e um modelo de sobrevida compartimentado (modelo de Markov).

O modelo de árvore de decisão foi selecionado para simular os possíveis resultados dos testes de diagnóstico em relação a cada mutação avaliada, bem como quais terapias de primeira linha (1L) seriam recomendadas de acordo com o guideline do NCCN (Figura 1) (National Comprehensive Cancer Network, 2019).
A quimioterapia combinada ao pembrolizumabe foi o pemetrexato e a carboplatina. A cisplatina não foi considerada na análise pelo fato de sua bula não ter indicação de uso para CPNPC (Accord Farmacêutica Ltda., 2016). O tratamento com gefitinibe foi considerado na análise como terapia-alvo de EGFR, devido ao fato de o seu estudo IPASS ser o único a avaliar a sobrevida livre de progressão (SLP) e a sobrevida global (SG) em pacientes com CPNPC tanto mutados quanto selvagens para EGFR (Mok et al., 2009; Fukuoka et al., 2011).

\section{Modelo de Markov}

O modelo de Markov foi usado com o intuito de acompanhar os pacientes que receberam o tratamento após o direcionamento do diagnóstico. O modelo de sobrevida foi particionado em três estados de saúde: SLP, progressão e morte (Figura 2). Assumiram-se ciclos mensais de transição entre os estados de saúde.

Tabela 3. Sensibilidade e especificidade das metodologias de diagnóstico avaliadas

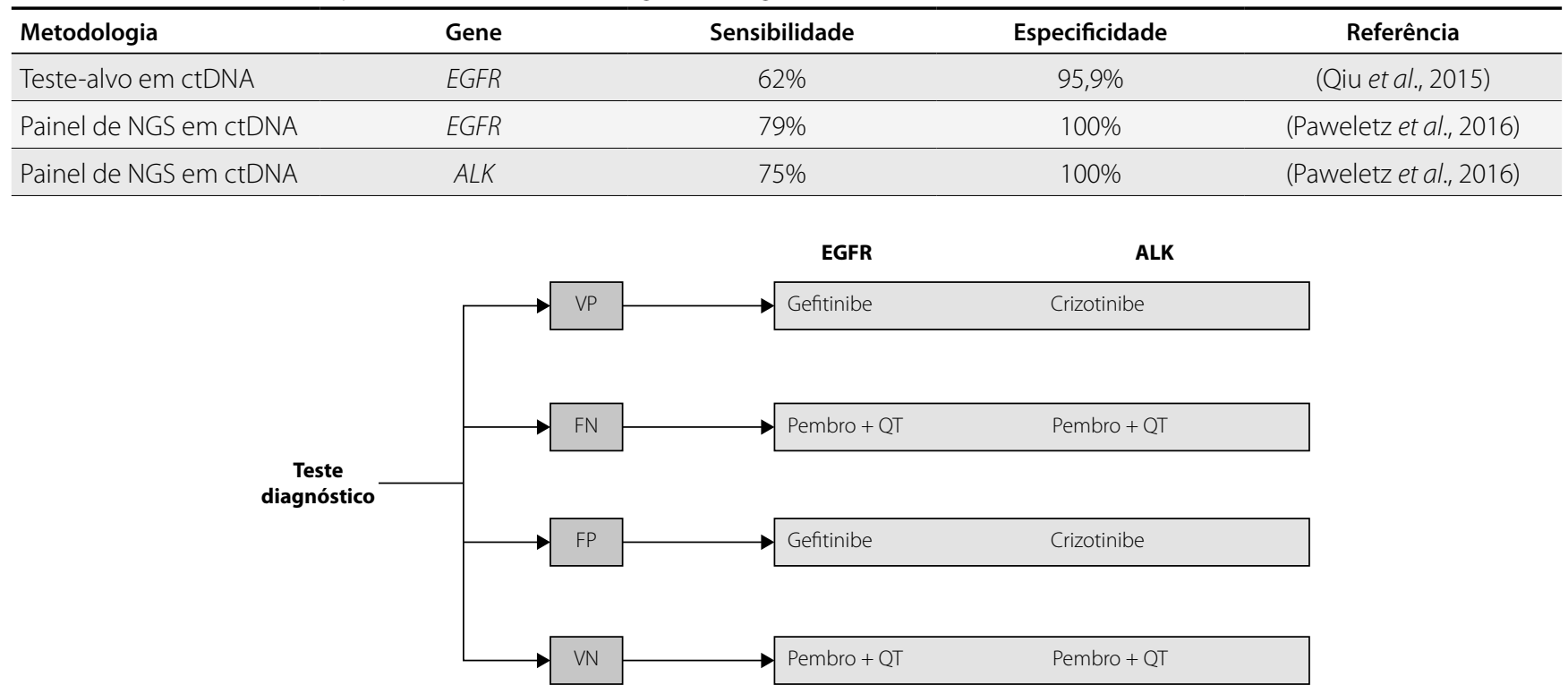

Figura 1. Modelo de árvore decisão.

VP: verdadeiro-positivo; FN: falso-negativo; FP: falso-positivo; VN: verdadeiro-negativo; QT: quimioterapia; Pembro: pembrolizumabe.

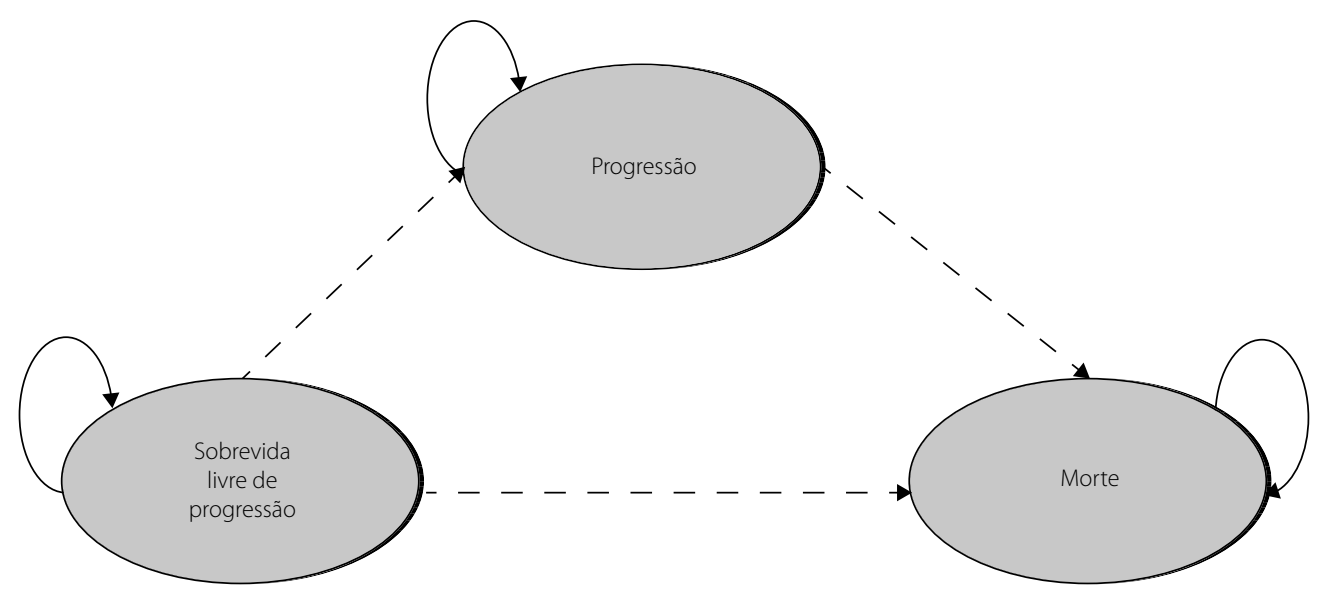

Figura 2. Modelo de Markov. 


\section{Probabilidade de transição}

As probabilidades de transição foram extraídas, quando possível, dos seus estudos pivotais. Os dados de eficácia foram extraídos dos estudos representados na Tabela 4.

O horizonte de tempo da análise foi de um ano, uma vez que nenhuma das terapias utilizadas apresentou duração de tratamento superior a esse período (Tabela 5) (Mok et al., 2009; Solomon et al., 2014; Gandhi et al., 2018).

As curvas foram parametrizadas com base no melhor ajuste dos dados, conforme a Tabela 6.

Evidências têm demonstrado que os pacientes com CPNPC e presença de alterações gênicas nos genes EGFR ou ALK apresentam benefício clínico limitado para as imunoterapias anti-PD-1 e anti-PD-L1, comparado aos pacientes selvagens para essas alterações (Gainor et al., 2016). O estudo KEYNOTE-010 demonstrou que os pacientes com CPNPC e presença de mutações em EGFR que foram tratados com pembrolizumabe não apresentaram diferença estatisticamente significativa para os desfechos de SG [hazzard ratio (HR): 0,88; intervalo de confiança (IC) de 95\%: 0,45 a 1,70) e SLP (HR: 1,79; IC de 95\%: 0,94 a 3,42) em relação aos pacientes tratados com quimioterapia (Herbst et al., 2016). Dessa forma, o modelo assumiu a premissa de que os pacientes com CPNPC e presença de alterações genômicas em EGFR ou ALK tratados com pembrolizumabe + quimioterapia não apresentaram di- ferença na SG e SLP em relação ao braço da quimioterapia dos seus respectivos estudos IPASS (Mok et al., 2009; Fukuoka et al., 2011) e Profile 1014 (Solomon et al., 2014, 2018).

\section{Dados epidemiológicos}

A prevalência das alterações nos genes EGFR e ALK em pacientes com CPNPC foi retirada de estudos brasileiros, sendo 25,5\% e 4\%, respectivamente (Pontes et al., 2014; Gomes et al., 2015).

\section{Uso de recursos e custos}

No estudo, foram considerados somente os custos médicos diretos [procedimento de diagnóstico, aquisição de medicamento, manejo dos eventos adversos (EAs) e progressão]. Os custos indiretos não foram incluídos.

\section{Procedimento de diagnóstico}

O custo unitário do procedimento de diagnóstico considerou somente o custo do painel de NGS em ctDNA. O custo com o procedimento de detecção de mutações no gene EGFR por ctDNA é arcado pelos programas de suporte ao paciente das indústrias farmacêuticas. Portanto, o seu custo não foi considerado. Já o procedimento relacionado ao painel de NGS, apesar de apresentar o código CBHPM (Classificação Brasileira Hierarquizada de Procedimentos Médicos), ainda não teve definida sua valoração (Associação Médica Brasileira, 2019b).

Tabela 4. Estudos considerados na análise

\begin{tabular}{ll}
\hline Terapias 1L & Estudos clínicos \\
\hline Gefitinibe & (Mok et al., 2009; Fukuoka et al., 2011) \\
\hline Crizotinibe (ALK) & (Solomon et al., 2014; Solomon et al., 2018) \\
\hline Pembro + QT & (Gandhi et al., 2018) \\
\hline
\end{tabular}

Tabela 5. Duração do uso das terapias de $1 \mathrm{~L}$

\begin{tabular}{ccc}
\hline Terapias 1L & Duração de uso (meses) & Estudos clínicos \\
\hline Gefitinibe & $5,6^{\mathrm{a}}$ & (Mok et al., 2009) \\
\hline Crizotinibe (ALK) & $10,9^{\mathrm{a}}$ & (Solomon et al., 2014) \\
\hline Pembro + QT & $7,4^{\mathrm{b}}$ & (Gandhi et al., 2018) \\
\hline
\end{tabular}

${ }^{\mathrm{a}}$ Mediana da duração de uso. ${ }^{\mathrm{b}}$ Média da duração de uso. TKI: inibidores da tirosina quinase; QT: quimioterapia.

Tabela 6. Distribuição paramétrica das curvas de sobrevida

\begin{tabular}{lcc} 
& Distribuição paramétrica (SLP) & Distribuição paramétrica (SG) \\
\hline Crizotinibe (ALK+) & Log-logistic & Weibull \\
\hline Gefitinibe (EGFR+) & Gompertz & Log-logistic \\
\hline Gefitinibe (EGFR-) & Log-logistic & Log-logistic \\
\hline Pembro + QT (EGFR- e ALK-) & Gompertz & Gompertz \\
\hline Pembro + QT EGFR+ & Log-logistic & Log-logistic \\
\hline Pembro + QT ALK+ & Gompertz & Weibull \\
\hline
\end{tabular}


Dessa forma, foi realizada uma pesquisa de mercado com o intuito de levantar o custo dos painéis de NGS nos principais laboratórios. Para o cálculo do preço médio, foram consideradas somente as metodologias que são realizadas em território nacional. Como não foi possível obter as informações do tamanho em pares de bases de todas as metodologias, a média foi calculada considerando todos os painéis de NGS levantados na pesquisa. O valor médio foi de R\$3.598,94 (Tabela 8).

\section{Tratamento medicamentoso}

Os custos unitários dos medicamentos foram obtidos da lista de preços da Câmara de Regulação do Mercado de Medicamentos (CMED) de julho de 2019, considerando o preço fábrica (PF 18\%) (Ministério da Saúde (Brasil), 2019). As posologias dos medicamentos foram baseadas nas suas respectivas bulas, listadas também na Tabela 8 . A posologia da bula da carboplatina foi ajustada com base no estudo KEYNOTE-189 (Gandhi et al., 2018), no qual faz o uso da terapia combinada ao pembrolizumabe a cada três semanas, em vez de quatro semanas, conforme recomendado pela bula (Glenmark Farmacêutica Ltda., 2017). A duração do tratamento considerada foi até a progressão da doença.

\section{Custo de progressão}

O custo do evento de progressão foi calculado como o somatório dos procedimentos relacionado à progressão, conforme a Tabela 10.

Tabela 7. Código CBHPM do procedimento de NGS

\begin{tabular}{ll}
\hline Código CBHPM & Procedimento \\
\hline $4.05 .03 .87-9$ & Captura/amplificação e subsequente sequenciamento de regiões \\
& genômicas DE 20 KILOBASES A 1 MEGABASE DE DNA TUMORAL \\
& PARA ANÁLISE DE MUTAÇÕES SOMÁTICAS por qualquer técnica de \\
& sequenciamento (Sanger ou qualquer forma de sequenciamento de nova \\
& geração - NGS) (valoração a ser definida) \\
\hline
\end{tabular}

Tabela 8. Custo unitário dos procedimentos de diagnóstico usado no modelo

\begin{tabular}{ll}
\hline Procedimento & Custo unitário \\
\hline EGFR ctDNA & $R \$ 0,00$ \\
\hline NGS ctDNA & $R \$ 3.598,94$ \\
\hline
\end{tabular}

Tabela 9. Custo e posologia dos medicamentos das terapias de $1 \mathrm{~L}$

\begin{tabular}{llll}
\hline Medicamentos & Posologia & Referência & Preço (PF 18\%) \\
\hline Carboplatina & $300 \mathrm{mg} / \mathrm{m}^{2}$ a cada 3 semanas & $\begin{array}{l}\text { (Gandhi et al., 2018) (Accord } \\
\text { Farmacêutica Ltda., 2017) }\end{array}$ & $\mathrm{R} \$ 1.219,87$ \\
\hline Crizotinibe & $250 \mathrm{mg} \mathrm{VO} \mathrm{2x/dia}$ & (Laboratórios Pfizer Ltda., 2018) & $\mathrm{R} \$ 31.007,30$ \\
\hline Gefitinibe & $250 \mathrm{mg} / \mathrm{dia}$ & $\begin{array}{l}\text { (AstraZeneca do Brasil Ltda., } \\
\text { 2018) }\end{array}$ & $\mathrm{R} \$ \mathbf{4 . 3 7 9 , 7 7}$ \\
\hline Pemetrexede & $500 \mathrm{mg} / \mathrm{m}^{2}$ a cada 3 semanas & $\begin{array}{l}\text { (Gandhi et al., 2018) (Glenmark } \\
\text { Farmacêutica Ltda., 2017) }\end{array}$ & $\mathrm{R}$ 4.865,89 \\
\hline Pembrolizumabe & $200 \mathrm{mg}$ a cada 3 semanas & $\begin{array}{l}\text { (Merck Sharp \& Dohme } \\
\text { Farmacêutica Ltda., 2018) }\end{array}$ & $\mathrm{R} \$ 15.135,19$ \\
\hline
\end{tabular}

VO: via oral.

Tabela 10. Custo dos procedimentos relacionados à progressão

\begin{tabular}{llll}
\hline Código & Descrição & Valor & Referência \\
\hline $4.07 .08 .12-8$ & PET dedicado oncológico & $R \$ 3.272,49$ & $\begin{array}{l}\text { (Associação Médica Brasileira, } \\
\text { 2019a) }\end{array}$ \\
\hline $4.10 .01 .22-2$ & $\begin{array}{l}\text { TC para PET dedicado } \\
\text { oncológico }\end{array}$ & $\mathrm{R} \$$ 1.419,67 & $\begin{array}{l}\text { (Associação Médica Brasileira, } \\
\text { 2019a) }\end{array}$ \\
\hline $1.01 .02 .02-7$ & Visita ou consulta médica & $R \$ 262,10$ & $\begin{array}{l}\text { (Associação Médica Brasileira, } \\
\text { 2019a) }\end{array}$ \\
\hline
\end{tabular}




\section{Custo unitário dos EAs}

Foi considerada, no estudo, a incidência dos EAs das terapias medicamentosas utilizadas em cada caso, bem como os seus custos. As frequências de ocorrência de cada evento foram obtidas a partir de seus estudos clínicos (Mok et al., 2009; Solomon et al., 2014; Gandhi et al., 2018). Somente os EAs de grau 3 ou maior foram incluídos no custo de tratamento (Tabela 11).

\section{Análise de sensibilidade}

Para as avaliações econômicas que suportam as tomadas de decisões, são fundamentais a quantificação das incertezas envolvidas nos seus resultados e a identificação das variáveis que mais afetam essas incertezas. Análise de sensibilidade univariada (ASU) e probabilística foi realizada para o desfecho de SLP.

Para a ASU, os parâmetros foram variados em $\pm 10 \%$. Em relação à análise de sensibilidade probabilística, foram realizadas 1.000 iterações, e os parâmetros foram variados conforme a Tabela 12.

\section{Resultados}

Os resultados de custo-efetividade do uso do painel de NGS em ctDNA em comparação com o teste de EGFR em ctDNA podem ser vistos na Tabela 12. O uso do painel de NGS em

Tabela 11. Custo unitário dos eventos adversos

\begin{tabular}{|c|c|}
\hline Evento & Custo por evento \\
\hline Aminotransferases elevadas & $R \$ 489,16$ \\
\hline Anemia & $R \$ 1.255,95$ \\
\hline Artralgia & $R \$ 325,52$ \\
\hline Astenia & $R \$ 313,92$ \\
\hline Constipação & $\mathrm{R} \$ 514,64$ \\
\hline Diarreia & $\mathrm{R} \$ 1.579,76$ \\
\hline Dificuldades visuais & $R \$ 250,60$ \\
\hline Dispneia & $R \$ 1.661,29$ \\
\hline Dor de cabeça & $\mathrm{R} \$ 357,49$ \\
\hline Edema periférico & $\mathrm{R} \$ 314,90$ \\
\hline Estomatite & $R \$ 1.563,92$ \\
\hline Fadiga & $R \$ 313,92$ \\
\hline Febre & $R \$ 1.567,73$ \\
\hline Leucopenia & $R \$ 4.823,96$ \\
\hline Mialgia & $R \$ 357,49$ \\
\hline Náusea & $\mathrm{R} \$ 1.584,98$ \\
\hline Neuropatia & $\mathrm{R} \$ 1.632,83$ \\
\hline Neutropenia & $\mathrm{R} \$ 8.318,72$ \\
\hline Perda de apetite & $R \$ 5.356,90$ \\
\hline Rash cutâneo & $R \$ 316,71$ \\
\hline Trombocitopenia & $R \$ 1.117,34$ \\
\hline Vômito & $\mathrm{R} \$ 1.589,22$ \\
\hline
\end{tabular}

ctDNA demonstrou economia de - $R \$ 2.076,35$ e aumento de SLP e SG de 0,271 e 0,0615 meses por paciente em um ano, respectivamente, comparado ao teste de EGFR em ctDNA. O custo do painel de NGS em ctDNA representa 0,99\% do custo total do paciente na primeira linha de tratamento em um ano.

\section{Análise de sensibilidade univariada}

Os resultados da análise de sensibilidade estão representados no diagrama de tornado apresentado na Figura 3.

Os resultados da ASU demonstraram que, mesmo variando os parâmetros do modelo, o painel de NGS em ctDNA continua sendo uma alternativa econômica em relação ao teste de EGFR em ctDNA. Os parâmetros que apresentaram maior influência na RCEl foram: o custo do pembrolizumabe, o custo do crizotinibe e a curva paramétrica de pembrolizumabe mais quimioterapia (SLP) entre casos ALK+.

Tabela 12. Distribuição de probabilidade para cada parâmetro avaliado na análise de sensibilidade probabilística

\begin{tabular}{l}
\hline Parâmetro $\quad$ Distribuição \\
\hline Custo unitário e uso de \\
recursos \\
- Custo unitário - \\
pembrolizumabe \\
- Custo unitário - crizotinibe Gama \\
- Custo unitário - gefitinibe \\
- Custo unitário - painel de \\
NGS ctDNA \\
- Custo de progressão
\end{tabular}

\section{Prevalência}

- Prevalência de EGFR Beta

- Prevalência de ALK

\section{Função paramétrica}

- Curva paramétrica gefitinibe (SLP) EGFR -

- Curva paramétrica gefitinibe (SLP) EGFR +

- Curva paramétrica crizotinibe (SLP) ALK

- Curva paramétrica pembrolizumabe + QT (SLP)

- Parametric model pembro + QT (PFS) EGFR +

- Parametric model pembro + QT (PFS) ALK +

- Curva paramétrica gefitinibe (SG) EGFR -

- Curva paramétrica gefitinibe (SG) EGFR +

- Curva paramétrica crizotinibe(SG) ALK

- Curva paramétrica pembrolizumabe + QT (SG)

- Curva paramétrica pembro + QT (PFS) EGFR+

- Curva paramétrica pembro + QT (PFS) ALK + 
$-\mathbf{R} \mathbf{2 0 . 0 0 0}$

Custo unitário - pembrolizumabe

Custo unitário - crizotinibe

Curva paramétrica pembro + QT (SLP) ALK +

Curva paramétrica pembro + CT (SLP)

Curva paramétrica pembro + CT (SLP) EGFR+

Custo unitário - painel de NGS

Curva paramétrica crizotinibe (SLP) ALK

Curva paramétrica gefitinibe (SLP) EGFR +

Custo unitário - gefitinibe

Curva paramétrica gefitinibe (SLP) EGFR

Custo unitário de progressão

Curva paramétrica pembro + CT (SG)

Curva paramétrica pembro + CT (SG) ALK +

Curva paramétrica crizotinibe (SG) ALK

Curva paramétrica gefitinibe (SG) EGFR +

Curva paramétrica pembro + CT (SG) EGFR +

Curva paramétrica gefitinibe (SG) EGFR -

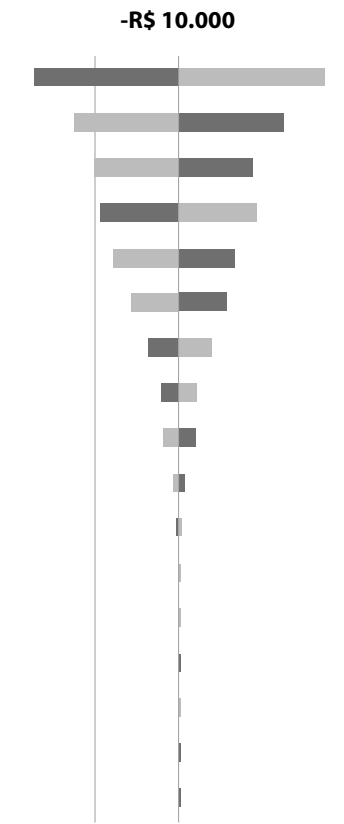

R\$ 0

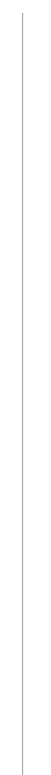

Figura 3. Diagrama de tornado.

Tabela 13. Resultados de custo-efetividade: painel NGS ctDNA versus teste EGFR ctDNA

\begin{tabular}{|c|c|c|c|}
\hline & NGS ctDNA & Teste EGFR ctDNA & Incremental \\
\hline \multicolumn{4}{|l|}{ Custos } \\
\hline Procedimento de diagnóstico & $R \$ 3.598,94$ & $\mathrm{R} \$ 0$ & $\mathrm{R} \$ 3.598,94$ \\
\hline Tratamento $1 \mathrm{~L}$ & $R \$ 355.718,91$ & $R \$ 361.246,38$ & $-R \$ 5.527,48$ \\
\hline Custo de progressão & $R \$ 2.257,20$ & $\mathrm{R} \$ 2.416,10$ & $-R \$ 158,90$ \\
\hline Eventos adversos & $\mathrm{R} \$ 1.233,03$ & $\mathrm{R} \$ 1.221,95$ & $R \$ 11,08$ \\
\hline Custo total & $R \$ 362.808,08$ & $\mathrm{R} \$ 364.884,44$ & $-R \$ 2.076,35$ \\
\hline \multicolumn{4}{|l|}{ Efetividade } \\
\hline SLP (meses) & 7,82 & 7,548 & 0,271 \\
\hline SG (meses) & 10,40 & 10,34 & 0,0615 \\
\hline RCEI (R\$/SLP) & & & $-\mathrm{R} \$ 7.652,56$ \\
\hline RCEI (R\$/SG) & & & $-\mathrm{R} \$ 33.742,14$ \\
\hline
\end{tabular}

\section{Análise de sensibilidade probabilística}

Os resultados da análise de sensibilidade probabilística estão representados na Tabela 14 e no gráfico da Figura 4, e demonstram que $80 \%$ das simulações se apresentaram no quadrante II (maior efetividade e menor custo), enquanto $20 \%$ das simulações se apresentaram no quadrante I (maior efetividade e maior custo).

\section{Discussão}

Estudos de modelagem econômica que avaliam o impacto dos testes diagnósticos nos desfechos clínicos e econômicos são escasssos na literatura. O presente estudo buscou mostrar a importância de um diagnóstico diferenciado por biópsia líquida, como o painel de NGS em ctDNA, em uma necessidade médica não atendida no CPNPC não escamoso e metastático. O uso do NGS em ctDNA possibilita direcionar os pacientes com material tecidual insuficiente de forma mais acertiva às terapias antineoplásicas, com base nas mutações oncogênicas avaliadas e nas terapias com indicação de bula no Brasil, recomendadas pelo guideline do NCCN.

Os gastos com imunoterapias podem ser mais bem direcionados com o uso do painel de NGS em ctDNA. Os pacientes com CPNPC e presença de alterações nos genes EGFR ou $A L K$ têm benefício limitado às terapias anti-PD1 e anti-PD-L1, 
Tabela 14. Resultados da análise de sensibilidade probabilística

\begin{tabular}{lccc}
\hline & Custo incremental & SLP (meses) incremental & RCEI por SLP (R\$) \\
\hline EGFR ctDNA & - & - & - \\
\hline NGS ctDNA & $-R \$ 2.069,95$ & 0,271 & $-R \$ 7.814,46$ \\
\hline
\end{tabular}

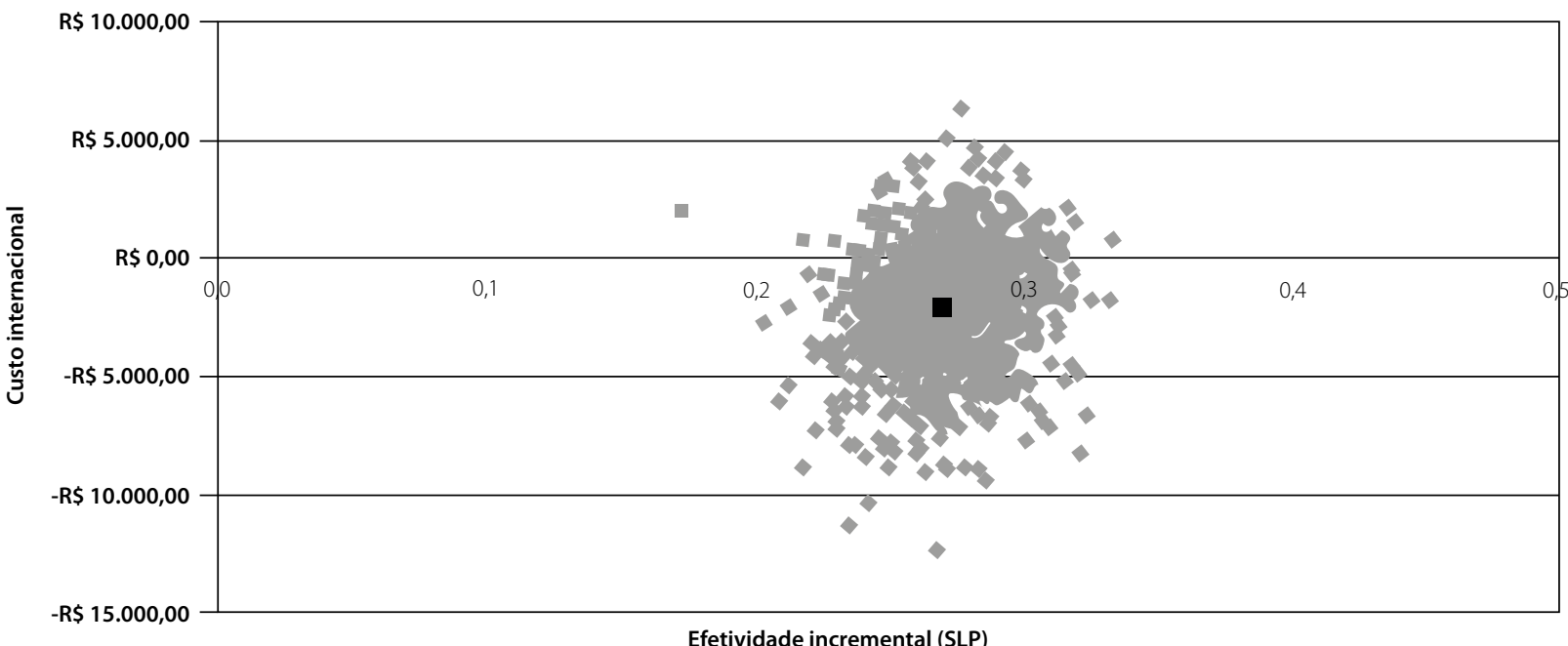

Figura 4. Resultados da análise de sensibilidade probabilística - Desfecho: SLP.

em detrimento aos casos selvagens (Gainor et al., 2016). Dessa forma, a avaliação dessas alterações utilizando painel de NGS em ctDNA possibilita reduzir a prescrição de imunoterapias para esses pacientes, por possibilitar o direcionamento destes para terapias-alvo.

O painel de NGS em ctDNA representa somente 0,99\% do custo total do paciente na primeira linha de tratamento em 1 ano. Mesmo considerando que as farmacêuticas arquem com o custo do teste de EGFR por ctDNA, o painel de NGS em ctDNA possibilita gerar economia para o SSS.

Vale ressaltar, como limitação, a ausência de um valor referência na tabela CBHPM para o painel de NGS. Como o valor não foi definido, foi considerado o valor de $\mathrm{R} \$ 3.598,94$ com base em uma pesquisa de mercado que levantou o valor dos painéis de NGS cobrados pelos laboratórios.

\section{Conclusão}

A presente análise concluiu que o painel de NGS em ctDNA é uma alternativa dominante (maior efetividade e menor custo) em relação ao teste de EGFR em ctDNA, na perspectiva do SSS. A economia de -R\$ 2 mil em um ano por paciente provém da redução da prescrição de imunoterapia na primeira linha de tratamento. A maior efetividade provém do ganho terapêutico com o direcionamento correto dos pacientes com mutações oncogênicas a suas terapias-alvo correspondentes.

\section{Referências bibliográficas}

Accord Farmacêutica Ltda. (2016) 'Cisplatina', pp. 1-17.

Accord Farmacêutica Ltda. (2017) 'Carboplatina', pp. 1-17.

Agência Nacional de Saúde Suplementar (ANS). Parecer Técnico n²7/ GEAS/GGRAS/DIPRO/2018 - Cobertura: medicamentos antineoplásicos orais. 2018. Available from: http://www.ans.gov.br/images/stories/ parecer_tecnico/uploads/parecer_tecnico/_PARECER_27-2018_ MEDICAMENTOS\%20ANTINEOPLSICOS\%20ORAIS_VERSO\%20 FINAL_27122017.pdf.

Associação Médica Brasileira (AMB). Classificação Brasileira Hierarquizada de Procedimentos Médicos (CBHPM). São Paulo: AMB; 2019a.

Associação Médica Brasileira (AMB). Resolução Normativa CNHM nº 038/2019. São Paulo: AMB; 2019b.

AstraZeneca do Brasil Ltda. (2018) Iressa (gefitinibe)', pp. 1-26.

Baldotto CSR, Julian GS, Mascarenhas E, Laloni MT, Azevedo CRAS, Kashiura $D$, et al. Padrões de tratamento, uso de recursos e custo do câncer de pulmão de não pequenas células avançado em instituições brasileiras privadas. J Bras Econ Saúde. 2018;10(2):86-106.

Barros JA, Valladares G, Faria AR, Fugita EM, Ruiz AP, Vianna AGD, et al. Diagnóstico precoce do câncer de pulmão: o grande desafio. Variáveis epidemiológicas e clínicas, estadiamento e tratamento. J Bras Pneumol. 2006;32(3):221-7.

Brambilla E, Travis WD, Colby TV, Corrin B, Shimosato Y. The new World Health Organization classification of lung tumours. Eur Respir J. 2001;18(6):1059-68.

Bray F, Ferlay J, Soerjomataram I, Siegel RL, Torre LA, Jemal A. Global cancer statistics 2018: GLOBOCAN estimates of incidence and mortality worldwide for 36 cancers in 185 countries. CA Cancer J Clin. 2018;68(6):394-424. 
Chouaid C, Dujon C, Do P, Monnet I, Madroszyk A, Le Caer H, et al. Feasibility and clinical impact of re-biopsy in advanced non small-cell lung cancer: A prospective multicenter study in a real-world setting (GFPC study 1201). Lung Cancer. 2014;86(2):170-3.

Diehl F, Schmidt K, Choti MA, Romans K, Goodman S, Li M, et al. Circulating mutant DNA to assess tumor dynamics. Nat Med. 2008;14(9):985-90.

Dive C, Brady G. SnapShot: Circulating Tumor Cells. Cell. 2017;168(4):742-742. e1.

Fukuoka M, Wu YL, Thongprasert S, Sunpaweravong P, Leong SS, Sriuranpong $\mathrm{V}$, et al. Biomarker analyses and final overall survival results from a phase III, randomized, open-label, first-line study of gefitinib versus carboplatin/paclitaxel in clinically selected patients with advanced non-small-cell lung cancer in Asia (IPASS). J Clin Oncol. 2011;29(21):2866-74.

Gainor JF, Shaw AT, Sequist LV, Fu X, Azzoli CG, Piotrowska Z, et al. EGFR Mutations and ALK Rearrangements Are Associated with Low Response Rates to PD-1 Pathway Blockade in Non-Small Cell Lung Cancer: A Retrospective Analysis. Clin Cancer Res. 2016;22(18):4585-93.

Gandhi L, Rodríguez-Abreu D, Gadgeel S, Esteban E, Felip E, De Angelis F, et al.; KEYNOTE-189 Investigators. Pembrolizumab plus Chemotherapy in Metastatic Non-Small-Cell Lung Cancer. N Engl J Med. 2018;378(22):2078-92.

Glenmark Farmacêutica Ltda. (2017) 'Pemetrexede', pp. 1-24. doi: 10.1360/ zd-2013-43-6-1064.

Gomes JR, Amarante MPF, D'Alpino R, Moreira RB, Sousa TTS, Lino AR, et al. Mutation profile in non-small cell lung cancer: Analysis of a Brazilian population. J Clin Oncol. 2015;33(15_Suppl):e19115.

Han X, Wang J, Sun Y. Circulating Tumor DNA as Biomarkers for Cancer Detection. Genomics Proteomics Bioinformatics. 2017;15(2):59-72.

Herbst RS, Baas P, Kim DW, Felip E, Pérez-Gracia JL, Han JY, et al. Pembrolizumab versus docetaxel for previously treated, PD-L1-positive, advanced non-small-cell lung cancer (KEYNOTE-010): a randomised controlled trial. Lancet. 2016;387(10027):1540-50.

Hong B, Zu Y. Detecting circulating tumor cells: current challenges and new trends. Theranostics. 2013;3(6):377-94.

Inca - Instituto Nacional de Câncer José Alencar Gomes da Silva. Estimativa 2018 - Incidência de câncer no Brasil. 2017. Available from: http:// www1.inca.gov.br/estimativa/2018/index.asp.

Laboratórios Pfizer Ltda. (2018) 'Xalkori (crizotinibe)', p. p.1-30.

Liu L, Liu H, Shao D, Liu Z, Wang J, Deng Q, et al. Development and clinical validation of a circulating tumor DNA test for the identification of clinically actionable mutations in nonsmall cell lung cancer. Genes Chromosomes Cancer. 2018;57(4):211-20.

Merck Sharp \& Dohme Farmacêutica Ltda. (2018) 'KEYTRUDA (pembrolizumabe) [bula]', p. p.1-32.

Ministério da Saúde (Brasil) (2019). Agência Nacional de Vigilância Sanitária (Anvisa). Câmara de Regulação do Mercado de Medicamentos (CMED). Available from: http://portal.anvisa.gov.br/cmed.

Mok TS, Wu YL, Thongprasert S, Yang CH, Chu DT, Saijo N, et al. Gefitinib or carboplatin-paclitaxel in pulmonary adenocarcinoma. N Engl J Med. 2009;361(10):947-57.
Müller JN, Falk M, Talwar J, Neemann N, Mariotti E, Bertrand M, et al. Concordance between Comprehensive Cancer Genome Profiling in Plasma and Tumor Specimens. J Thorac Oncol. 2017;12(10):1503-11.

National Comprehensive Cancer Network. NCCN Clinical Practice Guidelines in Oncology Version 5.2019 - Non-Small Cell Lung Cancer. NCCN Guideline. 2019.

Navada S, Lai P, Schwartz AG, Kalemkerian GP. Temporal trends in small cell lung cancer: Analysis of the national Surveillance, Epidemiology, and End-Results (SEER) database. J Clin Oncol. 2006;24(18_Suppl):7082.

Paweletz CP, Sacher AG, Raymond CK, Alden RS, O'Connell A, Mach SL, et al. Bias-Corrected Targeted Next-Generation Sequencing for Rapid, Multiplexed Detection of Actionable Alterations in Cell-Free DNA from Advanced Lung Cancer Patients. Clin Cancer Res. 2016;22(4):915-22.

Plagnol V, Woodhouse S, Howarth K, Lensing S, Smith M, Epstein M, et al. Analytical validation of a next generation sequencing liquid biopsy assay for high sensitivity broad molecular profiling. PLoS One. 2018;13(3):e0193802.

Pontes LDB, Bacchi CE, Queiroga EM, Piha T, Miranda PA, Freire S, et al. EGFR mutation screening in non-small cell lung cancer: results from an access program in Brazil. J Clin Oncol. 2014;32(15_Suppl):1526.

Qiu M, Wang J, Xu Y, Ding X, Li M, Jiang F, et al. Circulating tumor DNA is effective for the detection of EGFR mutation in non-small cell lung cancer: a meta-analysis. Cancer Epidemiol Biomarkers Prev. 2015;24(1):206-12.

Rolfo C, Mack PC, Scagliotti GV, Baas P, Barlesi F, Bivona TG, et al. Liquid Biopsy for Advanced Non-Small Cell Lung Cancer (NSCLC): A Statement Paper from the IASLC. J Thorac Oncol. 2018;13(9):1248-68.

Sher T, Dy GK, Adjei AA. Small cell lung cancer. Mayo Clin Proc. 2008;83(3):355-67.

Solomon BJ, et al. First-Line Crizotinib versus Chemotherapy in ALK -Positive Lung Cancer. N Engl J Med. 2014.

Solomon BJ, Kim DW, Wu YL, Nakagawa K, Mekhail T, Felip E, et al. Final Overall Survival Analysis From a Study Comparing First-Line Crizotinib Versus Chemotherapy in ALK-Mutation-Positive Non-Small-Cell Lung Cancer. J Clin Oncol. 2018;36(22):2251-8.

Thompson JC, Yee SS, Troxel AB, Savitch SL, Fan R, Balli D, et al. Detection of Therapeutically Targetable Driver and Resistance Mutations in Lung Cancer Patients by Next-Generation Sequencing of Cell-Free Circulating Tumor DNA. Clin Cancer Res. 2016;22(23):5772-82.

Travis WD, Brambilla E, Noguchi M, Nicholson AG, Geisinger KR, Yatabe $Y$, et al. International Association for the Study of Lung Cancer/ American Thoracic Society/European Respiratory Society International Multidisciplinary Classification of Lung Adenocarcinoma. J Thorac Oncol. 2011;6(2):244-85.

Travis WD, Brambilla E, Riely GJ. New pathologic classification of lung cancer: relevance for clinical practice and clinical trials. J Clin Oncol. 2013;31(8):992-1001. 